\title{
Absolute Voltage Amplification Using Dynamic Feedback Control
}

\author{
Paul C. de Jong and Gerard C. M. Meijer, Member, IEEE
}

\begin{abstract}
A new principle of voltage amplification that is insensitive to drift and aging effects of the feedback resistors is presented. The effect of mismatch of these resistors is reduced to a second-order effect which results in a total gain error of less than $5 \times 10^{-6}$ without the need for any calibration. Moreover, the long-term stability of the whole amplifier is guaranteed by the design when used as a chopper amplifier.
\end{abstract}

Index Terms-Absolute, amplification, chopper amplifier, chopping, dynamic feedback control, dynamic element matching, guaranteed long-term stability, $1 / f$ noise.

\section{INTRODUCTION}

$\mathbf{N}$ OWADAYS, the most accurate amplifiers are realized by applying negative feedback (by means of passive elements) around an active gain stage [1]. The low-frequency accuracy of the systems' input-output transfer function then is limited by the matching properties of these feedback elements. Even when laser-trimming techniques are used to achieve the desired accuracy, long-term stability is not guaranteed due to aging effects. In this paper, we present a novel technique to achieve gain stability without the need for accurate and stable feedback elements by using dynamic element matching [2]. We will present a high-precision voltage amplifier with a typical gain error of less than $5 \times 10^{-6}$ while calibration is superfluous and long-term stability is guaranteed by the design.

\section{Dynamic FeEdBack Control}

Fig. 1 shows an example of dynamic feedback control. The resistors $R_{1}$ and $R_{2}$, which are roughly equal, comprise the dynamic feedback of the amplifier. Their exchanging is controlled by a digital control block. During clock cycle "1" [Fig. 1(a)], the output voltage equals

$$
V_{\text {out }, 1}=\left(1+\frac{R_{2}}{R_{1}}\right) V_{\text {in }}
$$

Next, these resistors take each other's place [Fig. 1(b)], so

$$
V_{\text {out }, 2}=\left(1+\frac{R_{1}}{R_{2}}\right) V_{\text {in }} \text {. }
$$

If we define $R_{1}=R_{0}(1+\varepsilon)$ and $R_{2}=R_{0}(1-\varepsilon)$, then the average gain $\bar{G}=1 / 2\left(V_{\text {out }, 1}+V_{\text {out }, 2}\right) / V_{\text {in }}$ over two

Manuscript received June 3, 1996. This work was supported by the Dutch Foundation of Technical Sciences (STW) project DEL 33.3250.

The authors are with the Department of Electrical Engineering, Electronics Research Laboratory, Delft University of Technology, 2628 CD Delft, The Netherlands (e-mail: p.c.deJong@et.tudelft.nl, URL: http://duteela.et.tudelft.nl).

Publisher Item Identifier S 0018-9456(97)06465-6.

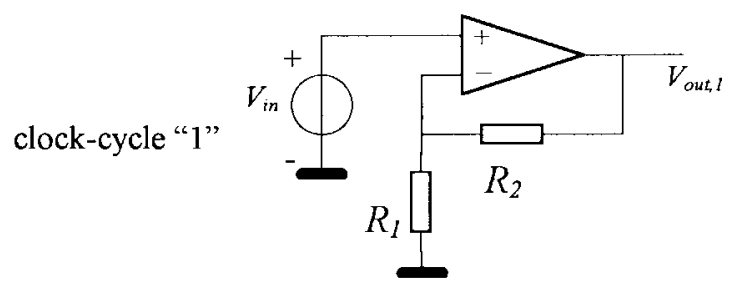

(a)

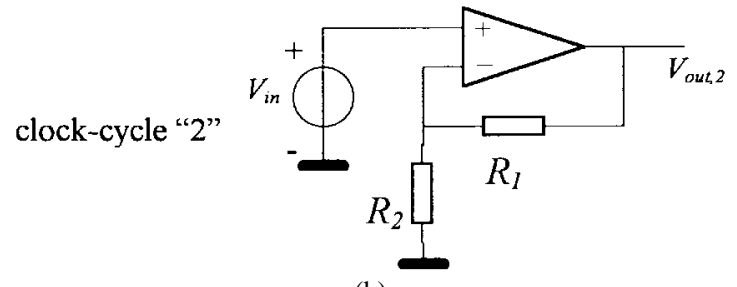

(b)

Fig. 1. A dynamic-feedback voltage amplifier during (a) clock-cycle "1" and (b) clock-cycle " 2 ".

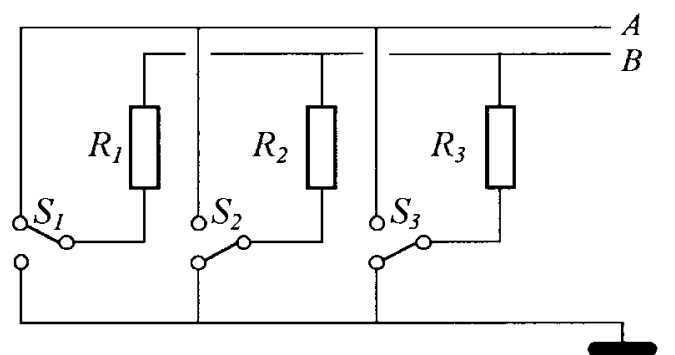

Fig. 2. Dynamic divider circuit, according to [2].

clock-cycles becomes

$$
\begin{aligned}
\bar{G} & =1+\frac{1+\varepsilon^{2}}{1-\varepsilon^{2}} \\
& \approx 2\left(1+\varepsilon^{2}\right)
\end{aligned}
$$

when $\varepsilon \ll 1$. So, resistor mismatches will be reduced to a second-order effect.

In order to set a gain $N$, we will need $N$ resistors. These resistors must be shifted along all possible positions of the feedback network.

Fig. 2 (after [2]) shows an example of a possible realization of such a network.

Switches will be necessary to realize the desired connections. Unfortunately, practical switches using MOS transistors have a significant voltage-dependent on-resistance due to the body-effect [3]. This will cause a gain error unless the 


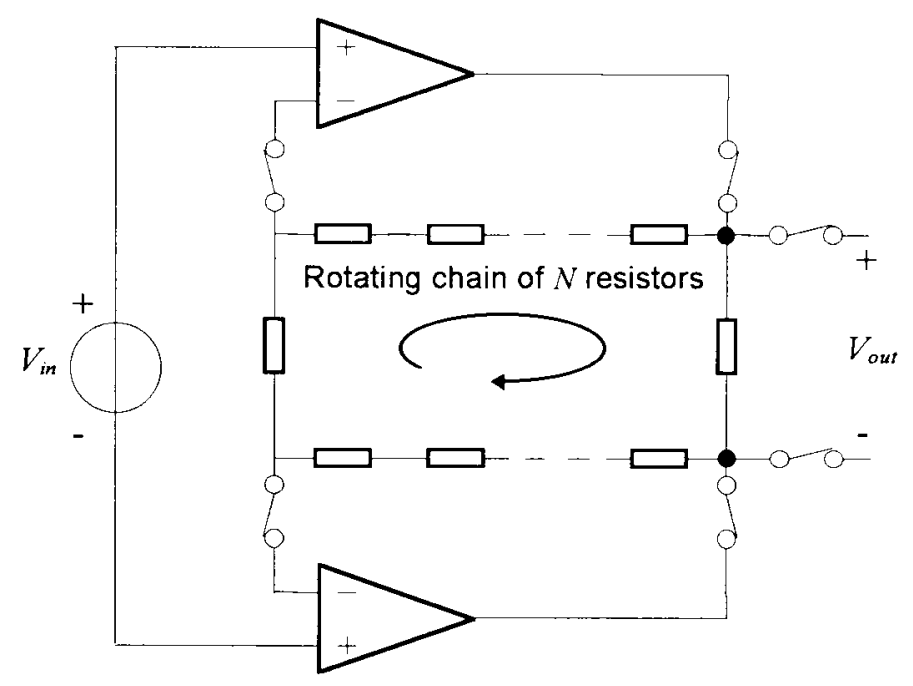

Fig. 3. The principle of the novel amplifier. The gain is not dependent of the on-resistance of the switches.

on-resistance of the switches is kept outside the signal transfer function of the amplifier.

Fig. 3 shows how this can be realized [4]. The resistors are connected as a chain that cyclically rotates between the two opamps. With each clock cycle, the chain rotates one position. When the chain consists of $N$ resistors, the average gain over $N$ clock cycles equals $N-1$ because there is one resistor connected as the load. Note that in this configuration $N$ is always even and $N \geq 4$.

The control of the resistor chain requires that there are six switches in between every two resistors. Two of these switches are necessary to connect to the negative input nodes of the opamps while two switches are necessary to connect to the output nodes of the opamps. Because the latter two switches conduct the signal current (Kelvin force), two more switches are necessary to sense the output voltage across the load resistor (Kelvin sense). Naturally, these switches should not conduct any current. So, the total amount of switches is $6 N$.

Fig. 4 shows a complete dynamic-controlled absolutevoltage amplifier with a voltage gain of seven. The addressing of the switches is performed by a cyclical counter and a decoder section. Each decoder output is connected to a group of six switches. For a certain clock-cycle, six switches are closed as indicated in Fig. 4. They form the conducting paths which are drawn as thick lines.

\section{ERROR ANALYSIS}

In this section, we will calculate the gain error of the dynamic-feedback amplifier in the configuration of Fig. 4. The gain $G$ of the voltage amplifier can be written as [1]

$$
G=\frac{V_{\text {out }}}{V_{\text {in }}}=\frac{1}{\beta} \cdot \frac{-A \beta}{1-A \beta}
$$

where $\beta$ is the attenuation factor of the feedback and $A$ the open-loop gain of the amplifier, respectively. The resulting gain error is thus related to the inaccuracy of the feedback and to the (closed) loop gain. For a high accuracy, $\beta$ must be well-defined while the loop gain $-A \beta$ must be (infinitely) large.

For calculation of the gain error, we assume that the amplifier consists of a chain of $N$ resistors. The average gain is defined as the average of $N$ successive gains

$$
\bar{G}=(N-1)(1+\Delta)
$$

where $\Delta$ represents the amplifiers' gain error.

\section{A. Resistive Feedback Related Errors}

Gain-errors can arise from effects that have a stochastic or systematic nature.

Resistor mismatch is a stochastic effect that contributes to the gain error. The resistor mismatch is represented by $\varepsilon$

$$
R_{i}=R_{0}\left(1+\varepsilon_{i}\right)
$$

where $i \in[1 \cdots N]$ and where $R_{0}$ equals the average resistor value of all $N$ resistors. Therefore,

$$
\sum_{i=1}^{N} \varepsilon_{i} \equiv 0
$$

Calculation of the gain error then yields

$$
\Delta=\frac{1}{N} \sum_{i=1}^{N} \varepsilon_{i}^{2}+\frac{2}{N(N-1)} \sum_{i=1}^{N / 2} \varepsilon_{i} \varepsilon_{i+N / 2}
$$

where it is assumed that $N$ is even and $N \geq 4$. Also, higherorder error-terms have been neglected.

The second term at the right-hand side of (8) is caused by the effect of connecting one resistor as a load resistor. For large gains, it will be negligible compared to the first term. As expected, (8) shows that the mismatches of the resistors will result in a second-order effect in the average gain of the voltage amplifier. Also note that the gain error is always positive.

Statistical simulations were performed in order to find a relation between the typical gain error as a function of the resistor mismatch. Fig. 5 shows the results of 10000 simulations on amplifiers with $G=7$. The resistor mismatch is modeled with an uniform distribution over an interval $\varepsilon \in$ $[-1 / 2 \%, 1 / 2 \%]($ standard deviation $=1 / \sqrt{12} \%)$ and with a Gaussian distribution $(\sigma=1 / \sqrt{12} \%)$, respectively. The gainerror distribution appears to be somewhat different and varies from Gamma to Gaussian. The gain-error distribution becomes more Gaussian when the gain increases. A good estimate of the average gain error is given by $\varepsilon^{2} / \sqrt{N}$.

Gain errors can also arise from effects that affect the resistor values in a systematical way.

The resistors must be independent of bias conditions, because bias conditions may change as we move along the resistor chain. This excludes certain resistor types. As this circuit has been implemented in a p-epi CMOS process, diffused or implanted n-type resistors are unsuitable because 


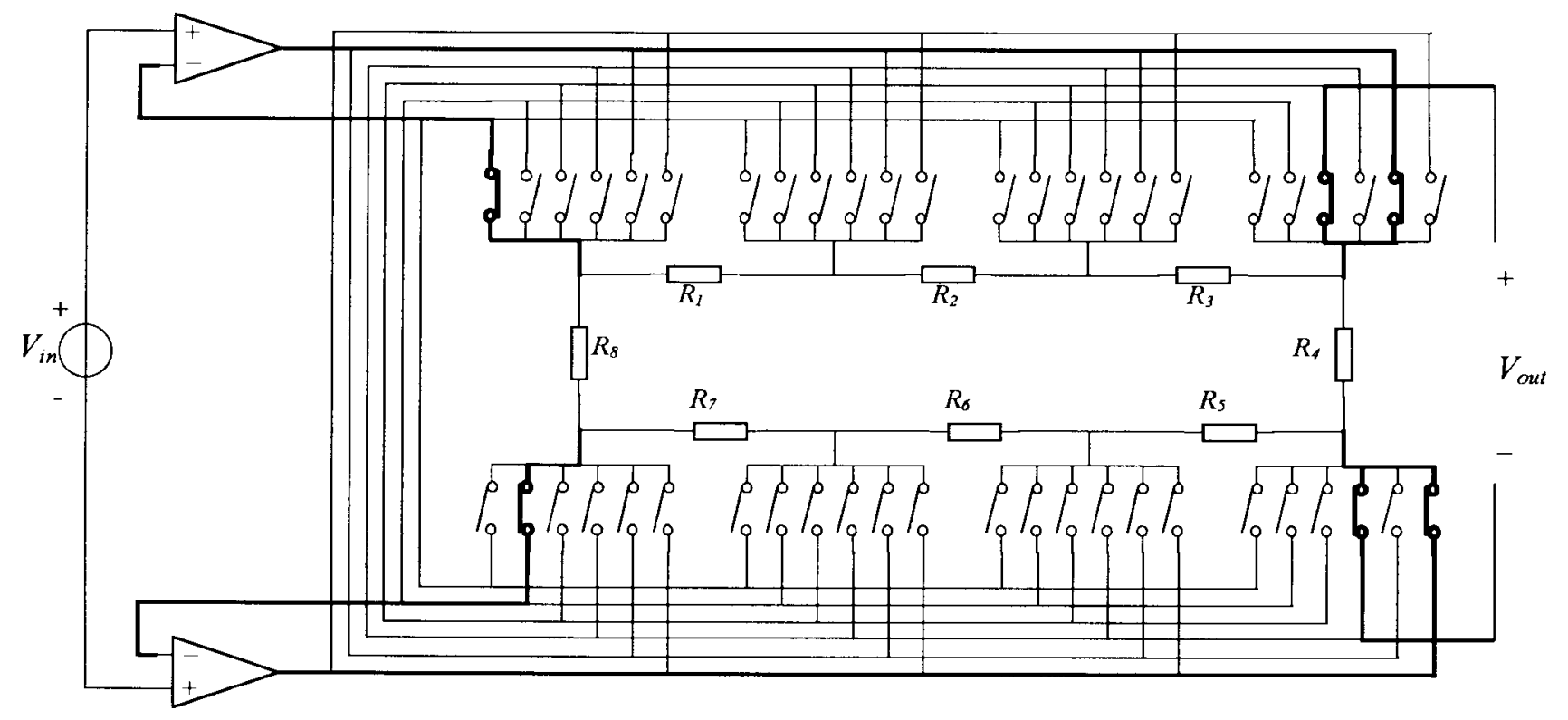

Fig. 4. The complete dynamic-controlled absolute voltage amplifier. The conducting paths are drawn as thick lines for a certain state of the addressing logic.

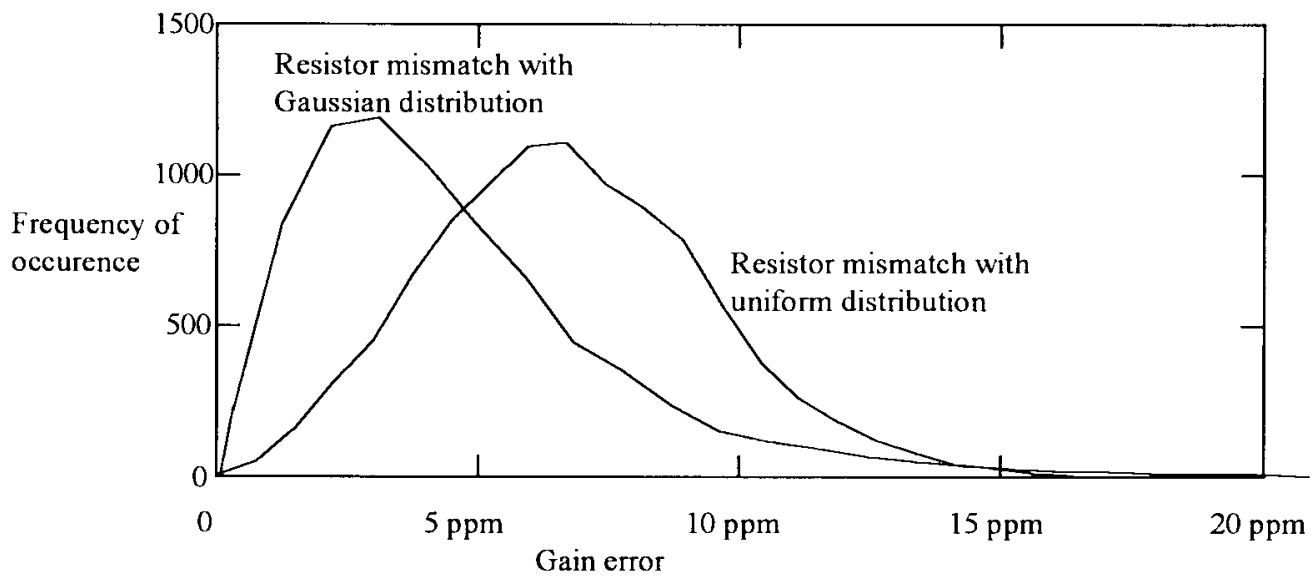

Fig. 5. Statistical simulation results of the gain error as a result of resistor mismatches (10000 simulations with $\sigma=1 / \sqrt{12} \%)$.

of their voltage dependency as a result of pinch-effects at the depletion region. This would create a gain error. Therefore, low-ohmic polysilicon resistors (on top of floating n-wells) are best suited to realize the feedback network.

High-ohmic polysilicon resistors show relatively poor $1 / f$ noise characteristics. In that case, the clock rate should be higher than the $1 / f$ noise corner frequency of the resistors. Otherwise, short-term drift would increase the gain error.

The resistor values are found as a tradeoff between noiseperformance and power consumption. Low-noise performance results in small resistor values. On the other hand, the current through the load resistor can be significant. When the powerconsumption is large enough to heat-up this load resistor, a second order error arises from the temperature dependency of this resistor.

\section{B. Opamp Related Errors}

Transient effects, when the resistor chain moves by one position, will always contribute to the gain error unless the amplifier is a part of a sampled data system. Therefore, the output voltage of the amplifier must be sampled after it has settled within several parts per million of its end-value. To first order, the open-loop gain of the opamps can be modeled as

$$
A=-\frac{A_{0}}{1+s \tau_{0}}
$$

where $A_{0}$ is the dc open-loop gain and $\tau_{0}$ the dominant low-frequency time-constant, respectively.

It can be shown that the finite value of $\tau_{0}$ only affects the transient effects and that in the quasistationary case, the gain error is only due to the value of $A_{0}$ and amounts to

$$
\Delta=\frac{-1}{1+A_{0} \beta} .
$$

So, in order to obtain an inaccuracy in the order of $10^{-6}$, the dc-gain $A_{0}$ must be in the order of $10^{6}$. 


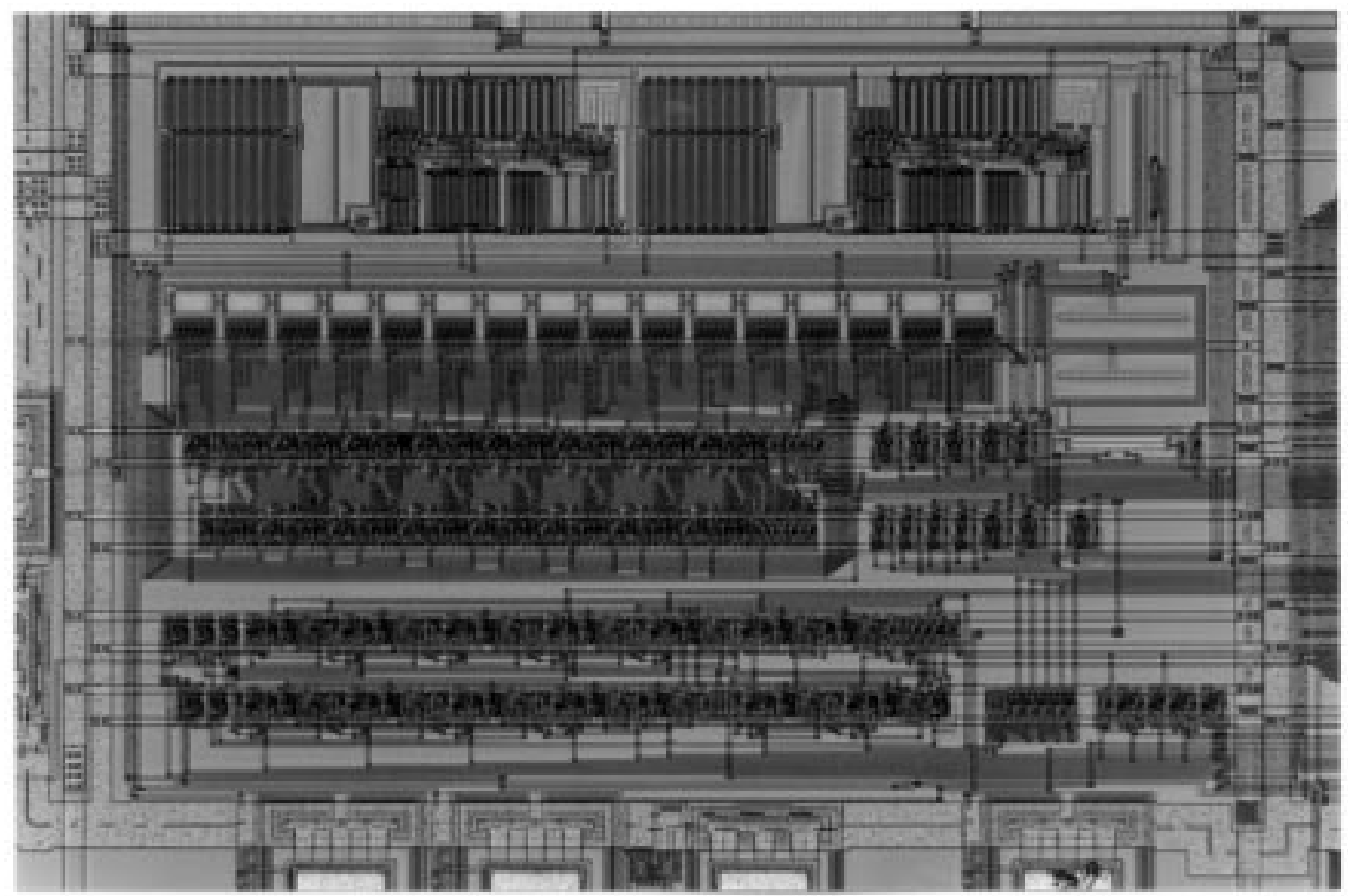

Fig. 6. Chip photograph of the absolute voltage amplifier $(G=15)$.

Remaining effects that limit the performance of the voltage amplifier are offset, $1 / f$ noise, and limited open-loop gain of the opamps.

Offset and $1 / f$ noise can sufficiently be attenuated by the use of chopping [5] or correlated double-sampling (CDS) [6]. We consider it as more robust to use chopping. Because the implementation of CDS requires sampling capacitors, it is likely that switches cause clock-feedthrough chargeinjection onto these sampling capacitors. Also, the timing of the switch control is critical. Especially at the output nodes, a large differential voltage can be expected which can easily result in signal-dependent clock-feedthrough [7]. Therefore, we implemented the amplifier with chopper stabilization. The chopper frequency runs at a frequency which is twice the clock frequency of the resistor chain. With the feedback network in a certain position, two samples of the output voltage will be taken, i.e., one per chop mode. Because the amplifier input signal alternates while the offset does not, subtraction of the two results yields elimination of the opamps' offset voltage.

The resulting clock frequency follows from a tradeoff between the $1 / f$-noise corner-frequency of the opamps and settling requirements as described previously. This results in practical clock frequencies in the range of $1 \mathrm{kHz}$ to $50 \mathrm{kHz}$. The absolute amplifier is used in an application [8], where the clock frequency is in the order of $8 \mathrm{kHz}$. So, a single resistor chain cycle (16 clock cycles) takes about $2 \mathrm{~ms}$.

\section{IMPLEMENTATION}

An absolute voltage amplifier with 16 resistors $(G=15)$ with chopper stabilization has been fabricated in a $0.7 \mu \mathrm{m}$
CMOS technology. A chip photo is shown in Fig. 6. The area of the core is $1100 \times 750 \mu \mathrm{m}^{2}$. From top-down, we can distinguish the two opamps, the resistor chain and the switches with their corresponding logic. The larger switches on the right-hand side form the chopper circuits. When compared to a common instrumentation amplifier, the logic circuits and switches increase the core area by $60 \%$.

\section{MEASUREMENT Results}

In order to measure the resulting gain error, we performed quasistatic measurements. The input of the amplifier is a resistive Wheatstone bridge with an imbalance such that the amplifier output voltage is approximately equal to the bridge supply voltage. The automated measurement setup measures the four bridge resistors in every position of the feedback network. The bridge supply voltage and the amplifier output voltage will be measured twice-one measurement per chop mode. Sampling is performed by a digital voltmeter and the data is stored digitally. From this data, the gain can easily be calculated. The applied clock frequency was very small $(1 \mathrm{~Hz})$ due to speed limitations of the measurement setup. This causes limited $1 / f$ noise suppression of the opamps. For measurement of the gain, this will result in an increase of the noise level of the measured voltages, but these low frequencies do not cause additional gain errors.

Fig. 7 shows the measured gain of the amplifier for each clock cycle. It can be clearly seen that the pattern repeats every 16 clock cycles. On the right-hand axis, the relative gain error is shown. It can be seen that the initial mismatching of the resistors is maximally $0.7 \%$. 


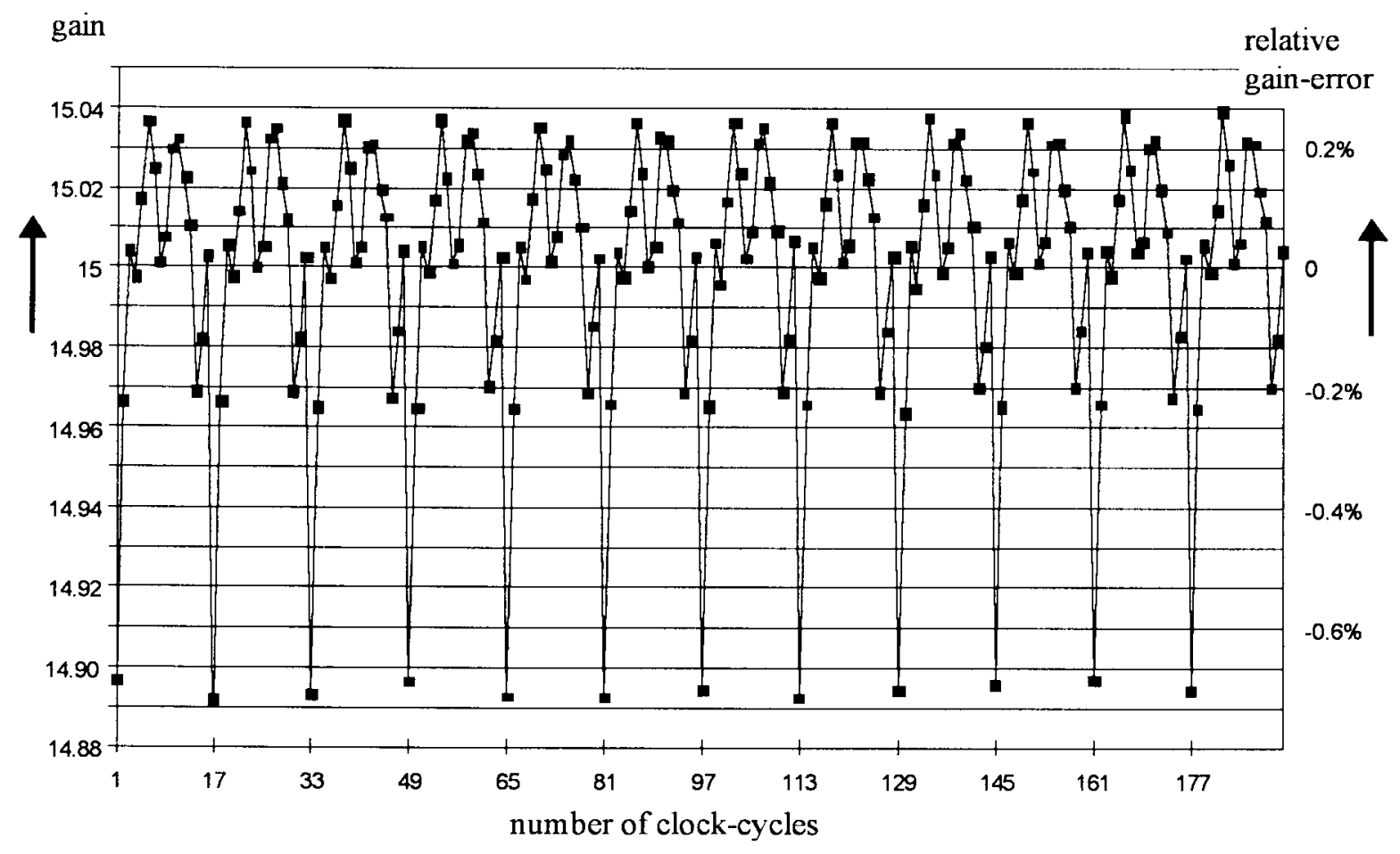

Fig. 7. The amplifier gain measured each clock cycle.

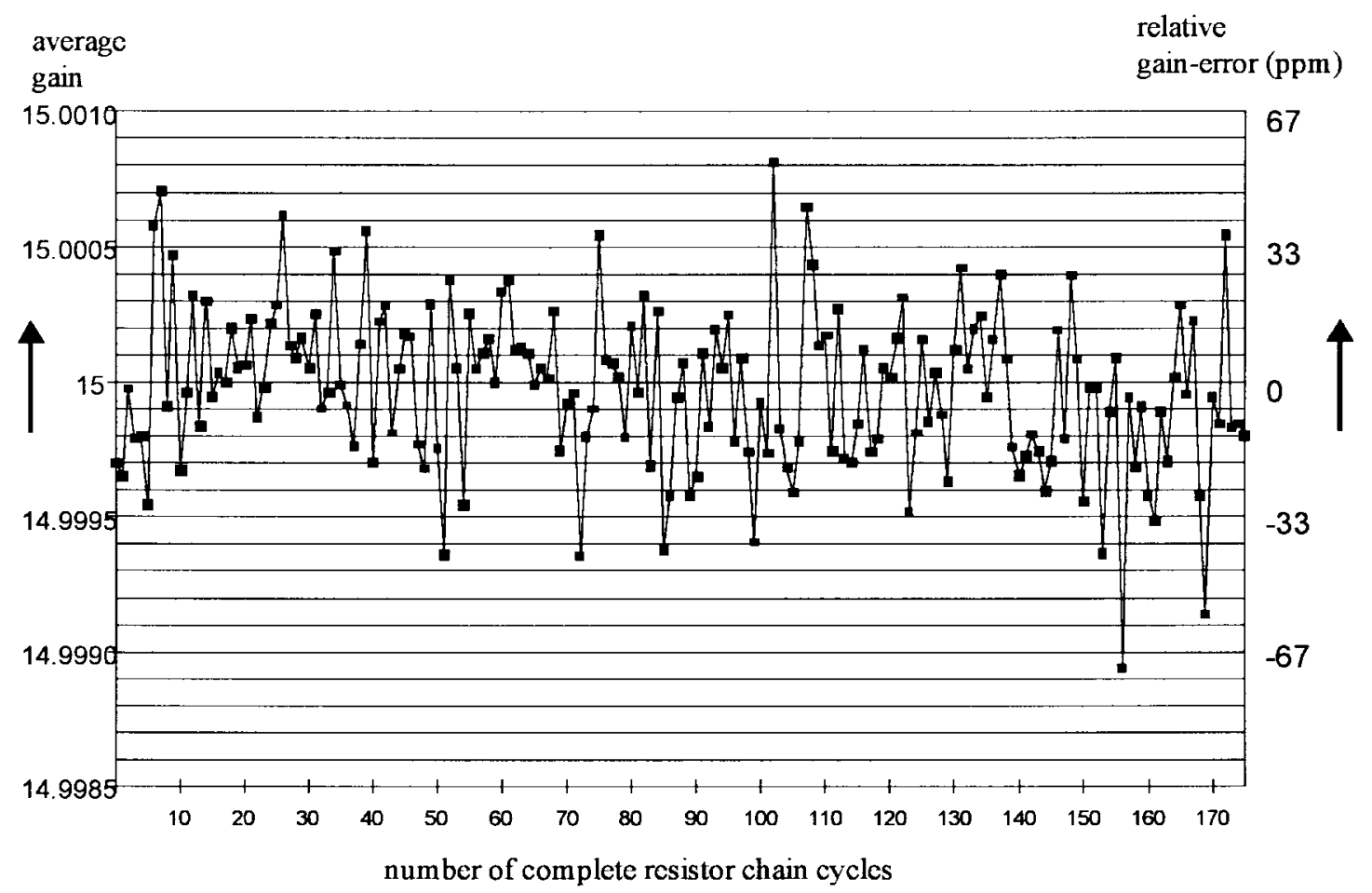

Fig. 8. The averaged gain as a function of the resistor chain cycle.

Fig. 8 shows 175 successive measurement results of the averaged gain over 16 clock cycles, i.e., 175 complete resistor chain cycles. The measurement setup requires extreme precision of the applied equipment. Again, the right-hand axis shows the relative gain error. A systematic gain error is not visible from this result, but is found to be less than $5 \times 10^{-6}$.
VI. CONCLUSION

An extremely accurate voltage amplifier for low-frequency applications has been designed, implemented, and tested. The use of dynamic feedback control results in a highly accurate and guaranteed long-term stable gain factor. In order to remove offset and $1 / f$ noise of the opamps, chopping is applied. A 
total systematic gain error of less than $5 \times 10^{-6}$ without the need for any calibration has been found.

\section{REFERENCES}

[1] E. H. Northolt, Design of High-Performance Negative Feedback Amplifiers. Amsterdam, The Netherlands: Elsevier, 1983.

[2] K. B. Klaassen, "Digitally controlled absolute voltage division," IEEE Trans. Instrum. Meas., vol. IM-24, pp. 106-112, June 1975.

[3] Y. P. Tsividis, Operation and Modeling of the MOS Transistor. New York: McGraw-Hill, 1987.

[4] P. C. de Jong, Dutch patent application 1.002.732, Mar. 1996.

[5] K. C. Hsieh, P. R. Gray, D. Senderowicz, and D. G. Messerschmitt, "A low-noise chopper-stabilized differential switched capacitor filtering technique," IEEE J. Solid-State Circuits, vol. 16, pp. 708-715, Dec. 1981.

[6] K. K. K. Lam and M. A. Copeland, "Noise cancellation switched capacitor (SC) filtering technique," Electron. Lett., vol. 19, pp. 810-811, Sept. 1983.

[7] B. J. Sheu and C. Hu, "Switch-induced error voltage on a switched capacitor," IEEE J. Solid-State Circuits, vol. 19, pp. 519-525, Aug. 1984.

[8] F. M. L. van der Goes and G. C. M. Meijer, "A novel low-cost and accurate multiple-purpose sensor interface with continuous autocalibration," in Proc. IMTC'96, Brussels, Belgium, June 4-6, 1996.

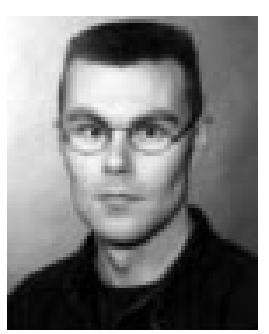

Paul C. de Jong was born in Nieuwkoop, The Netherlands, on May 13, 1967. In 1990, he received the ingenieurs (M.S.) degrees in electrical engineering from the Delft University of Technology, Delft, The Netherlands, where he is currently pursuing the Ph.D. degree.

In 1990, he joined Schlumberger, Clamart, France, and Houston, TX, where he was involved with high-temperature electronics for down-hole applications. In 1992, he joined the Laboratory of Electronics Research, Delft University of Technology. His research interests are in the field of electronic instrumentation, A/D converters, and high-temperature electronics.

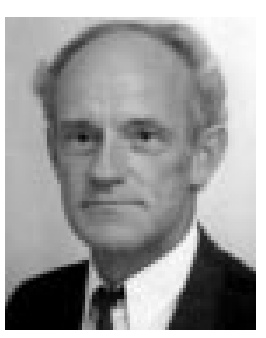

Gerard C. M. Meijer (M'94) was born in Wateringen, The Netherlands, on June 28, 1945. He received the ingenieurs (M.S.) and Ph.D. degrees in electrical engineering from the Delft University of Technology, Delft, The Netherlands, in 1972 and 1982, respectively.

Since 1972, he has been with the Laboratory of Electronics Research, Delft University of Technology, Delft, The Netherland, where he is an Associate Professor, engaged in research and teaching on analog IC's. In 1984 and part-time from 1985 to 1987 he was involved in the development of industrial level gauges and temperature transducers. In 1996 he was one of the founders of Sensart, where he is a consultant in the field of sensor systems.

Dr. Meijer is a member of the Netherlands Society for Radio and Electronics. 